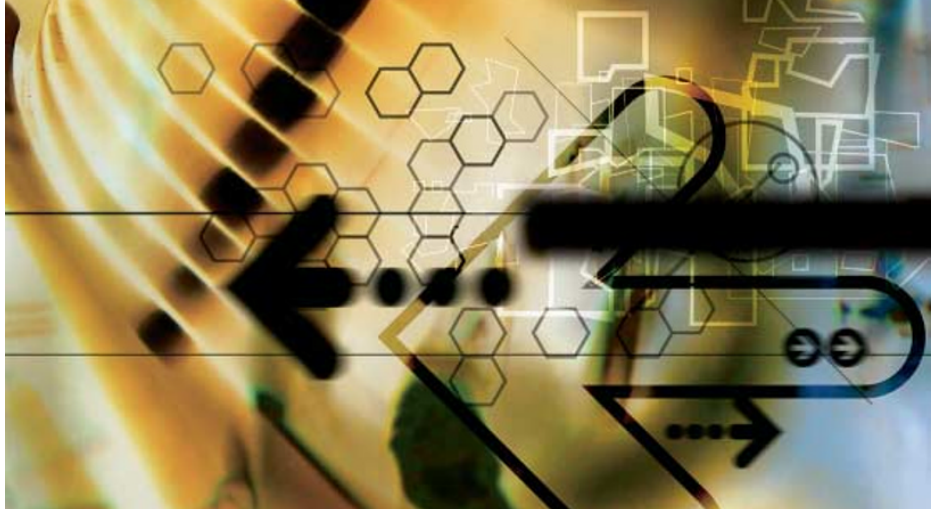

TUMORIGENESIS

\section{Working backwards}

Receptor tyrosine kinases of the EPH family are implicated in several human malignancies, but little is known about how they contribute to cancer development. Elena Pasquale and colleagues now show that EPHB4 promotes tumour angiogenesis by signalling through its membrane-bound ligand on endothelial cells.

EPHB4 is thought to be involved in breast cancer development, but there is contradictory evidence as to whether it has a positive or negative effect on tumour growth. To investigate this, the authors treated MDAMB-435 human breast cancer cells - which express EPHB4 - with a soluble form of the EPHB4 ligand, ephrin B2. Although the catalytic activity of the receptor was stimulated, cell growth was inhibited, indicating that EPHB4 kinase activity is not required for tumour progression.

However, as well as signalling through its kinase domain, EPHB4 can also transduce effects through binding of its ectodomain to ephrin B2 on adjacent cells. Ephrin B2 is a transmembrane protein with a cytoplasmic signalling domain that is activated on EPHB4 binding. Could this kinase-independent 'reverse signalling' by EPHB4 have a role in tumour growth? To test this, the authors transfected MDA-MB-435 cells with a kinase-defective form of EPHB4. Injection of these cells into mouse mammary fat pads resulted in the formation of tumours that grew significantly faster than those formed by control cells.

EPHB4 reverse signalling through ephrin B2 is involved in blood-vessel growth during development, indicating that the tumour-promoting activity of kinase-defective EPHB4 might result from increased angiogenesis. Consistent with this, tumours derived from MDA-MB435 cells expressing kinase-defective EPHB4 contained larger blood vessels and more blood than those derived from cells transfected with a control construct. In addition, ephrin B2 was shown to be expressed in blood vessels associated with MDAMB-435-derived tumours, indicating that EPHB4 on tumour cells might promote angiogenesis by interacting with ephrin B2 on endothelial cells.

Pasquale and colleagues showed that MDA-MB- 435 cells transfected with kinase-defective EPHB4 attracted human endothelial cells expressing ephrin B2 in a cell-migration assay. The same result was obtained using a solubilized form of the EPHB4 ectodomain, ruling out the possibility that another chemo-attractant expressed in the tumour cells was responsible for this effect. The ectodomain construct also promoted endothelial-cell invasion through a collagen gel, indicating that EPHB4 reverse signalling is sufficient to promote infiltration and vascularization of tumours by ephrin-B2-expressing endothelial cells.

This study provides a novel insight into mechanisms of tumour angiogenesis and new potential targets for anticancer therapy. How ephrin B2 signalling promotes endothelial-cell migration, and whether there is crosstalk with other angiogenic pathways, will be key questions for future studies.

Louisa Flintoft

(2) References and links ORIGINAL RESEARCH PAPER Noren, N. K.,

Lu, M., Freeman, A. L., Koolpe, M. \& Pasquale, E. B. Interplay between EphB4 on tumor cells and vascular ephrin-B2 regulates tumor growth. Proc Natl Acad. Sci. USA 101, 5583-5588 (2004)

\section{TRIAL WATCH}

\section{Anti-apoptosis drug shows promise}

Promising early clinical trial results of a targeted antiapoptosis agent, phenoxodiol (Marshall Edwards Inc), in patients with cancer were reported at the Annual Meeting of the American Association of Cancer Research (AACR) on 30th March 2004.

Sphingosine kinase regulates cell survival by signalling through the AKT pathway to activate the proteins X-linked inhibitor of apoptosis (XIAP) and FLICE inhibitory protein (FLIP), which block caspase activity, so preventing caspasetriggered apoptosis. In cancer cells, sphingosine-kinase activity is greatly increased. Phenoxodiol is a novel isoflavone and is thought to target a regulator of sphingosine kinase, so depriving the cell of XIAP and FLIP — the cancer cells then undergo apoptosis.

One Phase Ib/IIa trial being conducted in Australia and the United States has so far enrolled 24 patients with hormonerefractory prostate cancer and rising prostate-specific antigen (PSA) levels. The interim results reported by Graham Kelly in a late-breaking session at the AACR indicate that oral phenoxodiol is biologically active - a dose-dependent effect on PSA was seen. Six patients of twelve who were given the highest doses of the drug had a reduction in PSA of more than $50 \%$. No toxicity was reported. The next stage will be to test phenoxodiol in combination with a taxane in prostate cancer.

In a second Phase II study conducted at Yale University, phenoxodiol has shown activity as monotherapy in 40 patients with late-stage unresponsive ovarian cancer. On average, the patients have failed five different chemotherapy regimens. Ten of the forty patients responded after 6 weeks treatment, as measured by a fall or stabilization in blood levels of CA125 or improved clinical status. Again, the drug was well tolerated. Ten women who received phenoxodiol and whose disease had later progressed were then re-challenged with standard therapy paclitaxel, and eight of the ten responded with an average of a $64 \%$ decrease in CA125 levels. Four of these women had previously been considered to be resistant or refractory to paclitaxel, so these data indicate that phenoxodiol is a chemosensitizing agent. Tumour response has not yet been assessed.

In a third abstract, preclinical results were reported that supported the clinical observation that phenoxodiol can act as a chemosensitizer. The drug restored sensitivity of ovarian cancer cells to platinum agents and to the antimetabolite gemcitabine. Ovarian cancer cells showed a greater than 100 fold increase in sensitivity to chemotherapy after pretreatment with phenoxodiol.

The company is continuing to test phenoxodiol in clinical trials as first-line monotherapy for early-stage cancers, as first-line therapy for late-stage cancers and as second-line therapy as a chemosensitizer.

ORIGINAL RESEARCH PAPERS Davies, R. et al. Interim results of a phase lb/lla study of oral phenoxodiol in patients with late-stage, hormone-refractory prostate cancer. Proc. Am. Assoc. Cancer Res. abstract LB-214 (2004) | Rutherford, T. et al. Phenoxodiol phase lb/ll study in patients with recurrent ovarian cancer that are resistant to second line

chemotherapy. Proc. Am. Assoc. Cancer Res. abstract 4457 (2004) | Mor, G. et al. Phenoxodiol a chemosensitizer in taxotere-resistant ovarian cancer cells. Proc. Am. Assoc. Cancer Res. abstract 4885 (2004)

WEB SITE Marshall Edwards Inc: www.phenoxodiol.com 